\title{
A HOPF BIFURCATION IN SINGLE-LOOP POSITIVE-FEEDBACK SYSTEMS*
}

BY JAMES F. SELGRADE (North Carolina State University)

\begin{abstract}
This paper gives sufficient conditions for a Hopf bifurcation in a fivedimensional system of ordinary differential equations which provides a model for positive feedback in biochemical control circuits. These conditions only depend on the feedback function and its first and second derivative. The conditions are used to exhibit Hopf bifurcations for the Griffith equations and the Tyson-Othmer equations.
\end{abstract}

1. Introduction. Single-loop positive feedback in biochemical control circuits $[2,5,7]$ may be modeled using the $n$-dimensional system of ordinary differential equations

$$
\begin{aligned}
\dot{x}_{1} & =f\left(x_{n}\right)-\alpha_{1} x_{1} \\
\dot{x}_{i} & =x_{i-1}-\alpha_{i} x_{i}, \quad 2 \leq i \leq n,
\end{aligned}
$$

where $\alpha_{i}>0$ for $i=1, \ldots, n$, and $f$ is a bounded $C^{2}$ function with $f\left(x_{n}\right)>0$ and $f^{\prime}\left(x_{n}\right)>0$ for $x_{n}>0$. For the Griffith model [2] we have

$$
f\left(x_{n}\right)=x_{n}^{p} /\left(1+x_{n}^{p}\right)
$$

where $p$ is a positive integer. For the Tyson-Othmer model [7] we have

$$
f\left(x_{n}\right)=\left(1+x_{n}^{p}\right) /\left(K+x_{n}^{p}\right)
$$

where $p$ is a positive integer and $K>1$.

Let $x$ denote a point in $\mathbb{R}^{n}$ and $x_{i}$ denote its $i$ th component. The positive orthant in $\mathbb{R}^{n}$ is given by

$$
\mathscr{H} \equiv\left\{x \in \mathbb{R}^{n}: x_{i} \geq 0 \text { for all } 1 \leq i \leq n\right\} .
$$

In [6] we show that $\mathscr{H}$ is invariant under the positive-time solution flow of (1) and positive-time solutions in $\mathscr{H}$ are bounded. The critical points of (1) are situated on a half-line through the origin. If the critical points are finite in number and nondegenerate then they alternate between asymptotically stable and unstable, with orbits connecting adjacent critical points. The key to our analysis is that the off-diagonal terms in the linearization of (1) are nonnegative. Hence solutions to (1) preserve coordinatewise inequality in $\mathscr{H}[1,6]$. If $n=2$ or $n=3$ we show that the positive orbit of each point in $\mathscr{H}$ is asymptotic to a critical point. For higher dimensions, Hirsch [3] shows that the positive orbit of almost every point in $\mathscr{H}$ is asymptotic to the critical points. The stronger result which holds for $n=2$ and $n=3$ is not possible for $n \geq 5$. Here we derive sufficient conditions for a Hopf bifurcation with $n=5$ which depend only on $f, f^{\prime}$, and $f^{\prime \prime}$. We use these conditions to show Hopf bifurcations occur in the Griffith and Tyson-Othmer equations

\footnotetext{
* Received July 27, 1981.
} 
for certain values of the parameter $\alpha$. Hence, in these cases, (1) has an unstable periodic solution. Tyson and Othmer [7] suggest that a Hopf bifurcation may occur in their positive feedback model. Analogous results are true for $n>5$. But for $n=4$ there may be a critical point with one positive and one negative eigenvalue and two purely imaginary eigenvalues. The complex eigenvalues must remain on or to the left of the imaginary axis. The existence of periodic solutions is not clear in this case.

Henceforth, we assume $n=5$ and we take $\alpha_{i}=\alpha$ for all $1 \leq i \leq 5$ to simplify the computations. With $\alpha$ as parameter, (1) can be written in vector form as $\dot{x}=F_{\alpha}(x)$ where $x \in \mathbb{R}^{5}$. We give conditions which, when satisfied, guarantee that as $\alpha$ increases there is a critical point of $F_{\alpha}$ with a pair of eigenvalues crossing the imaginary axis with negative speed, i.e. the derivative of the real part of the eigenvalues with respect to $\alpha$ is negative. Thus a Hopf bifurcation occurs [4].

2. Results. For the critical points of (1) we need $f\left(x_{5}\right)=\alpha x_{1}, x_{1}=\alpha x_{2}, \ldots, x_{4}=\alpha x_{5}$. Thus we want $f\left(x_{5}\right)=\alpha^{5} x_{5}$. Hence define the function $g:[0, \infty) \times[0, \infty) \rightarrow \mathbb{R}$ by

$$
g(\alpha, c) \equiv f(c)-\alpha^{5} c .
$$

Note that $g(\alpha, 0) \geq 0$ and $g(\alpha, c) \rightarrow-\infty$ as $c \rightarrow \infty$ for all $\alpha>0$. For each $\alpha$, the critical points of $F_{\alpha}$ are in "1-1" correspondence with the solutions of $g(\alpha, c)=0$. The curve $g(\alpha, c)=0$ for the Griffith equations is sketched in Fig. 1. So the Griffith equations have one, two or three critical points depending on the value of $\alpha$.

The matrix $M_{\alpha}(c)$ for the linearized equations of (1) at a critical point with 5th coordinate $c$ is:

$$
M_{\alpha}(c)=\left(\begin{array}{ccccc}
-\alpha & 0 & 0 & 0 & f^{\prime}(c) \\
1 & -\alpha & 0 & 0 & 0 \\
0 & 1 & -\alpha & 0 & 0 \\
0 & 0 & 1 & -\alpha & 0 \\
0 & 0 & 0 & 1 & -\alpha
\end{array}\right)
$$

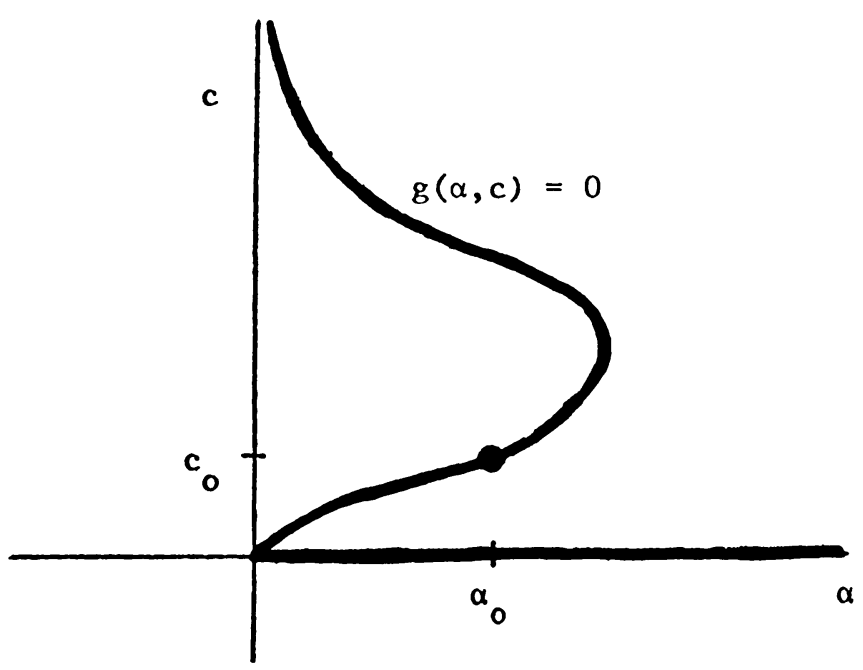

Fig. 1. 
If $A(c)$ denotes the $5 \times 5$ matrix with $f^{\prime}(c)$ in the upper right corner and 1's on the subdiagonal and zeroes elsewhere, then

$$
M_{\alpha}(c)=A(c)-\alpha I
$$

where $I$ is the $5 \times 5$ identity matrix. Clearly, the eigenvalues of $A(c)$ are the 5 roots of unity each multiplied by $\left(f^{\prime}(c)\right)^{1 / 5}$. The spectrum of $M_{\alpha}(c)$ is obtained by translating the spectrum of $A(c)$ to the left by an amount $\alpha$. The real part of the crucial eigenvalues of $M_{\alpha}(c)$ is given by the function $\lambda:(0, \infty) \times(0, \infty) \rightarrow \mathbb{R}$ defined by

$$
\lambda(\alpha, c) \equiv f^{\prime}(c)^{1 / 5} \cos (2 \pi / 5)-\alpha .
$$

For a nonzero critical point of $F_{\alpha}$ to have a pair of imaginary eigenvalues we need a solution to $\lambda(\alpha, c)=0$. Hence, for the possibility of a Hopf bifurcation in $\mathscr{H}$, we must find a solution $\left(\alpha_{0}, c_{0}\right), \alpha_{0} \neq 0$ and $c_{0} \neq 0$, to both the following equations:

$$
g(\alpha, c)=0, \quad \lambda(\alpha, c)=0 .
$$

For each solution $\left(\alpha_{0}, c_{0}\right)$ to $(4), \alpha_{0}$ is determined explicitly in terms of $c_{0}$ by

$$
\alpha_{0}\left(f\left(c_{0}\right) / c_{0}\right)^{1 / 5} .
$$

Define the function $\bar{\lambda}(\alpha, c)$ by

$$
\bar{\lambda}(\alpha, c) \equiv f^{\prime}(c) \cos ^{5}(2 \pi / 5)-\alpha^{5}
$$

and notice that $\left(\alpha_{0}, c_{0}\right)$ is a solution to (5) if and only if $\left(\alpha_{0}, c_{0}\right)$ is a solution to $\bar{\lambda}(\alpha, c)=0$. Let $k \equiv \cos ^{5}(2 \pi / 5)$ and define a function $h:(0, \infty) \rightarrow \mathbb{R}$ by

$$
h(c) \equiv k c f^{\prime}(c)-f(c) .
$$

Because of (6), we have that $\left(\alpha_{0}, c_{0}\right)$ is a solution to (4) and (5) if and only if $c_{0}$ is a solution to

$$
h(c)=0 .
$$

Assume $\left(\alpha_{0}, c_{0}\right)$ is a solution to (4) and (5). From (6) it follows that

$$
\left.\frac{d \alpha}{d c}\right|_{c_{0}}=\frac{c_{0} f^{\prime}\left(c_{0}\right)-f\left(c_{0}\right)}{5 \alpha_{0}^{4} c_{0}^{2}} .
$$

Since $k<1, c_{0} f^{\prime}\left(c_{0}\right)-f\left(c_{0}\right)>k c_{0} f^{\prime}\left(c_{0}\right)-f\left(c_{0}\right)=h\left(c_{0}\right)=0$. Thus $d \alpha / d c$ at $c_{0}$ is positive; let $c(\alpha)$ denote the inverse function of (6) defined in a neighborhood of $\alpha_{0}$. For the eigenvalues of $M_{\alpha}(c(\alpha))$ to be crossing the imaginary axis at $\alpha_{0}$ with nonzero speed, we need to show that

$$
\left.\frac{d \lambda}{d \alpha}(\alpha, c(\alpha))\right|_{\alpha_{0}}<0 .
$$

But the sign of $d \lambda / d \alpha$ at $\alpha_{0}$ is the same as the sign of $d \bar{\lambda} / d \alpha$ at $\alpha_{0}$. And the sign of $d \bar{\lambda} / d \alpha$ at $\alpha_{0}$ is the same as the sign of the derivative of $c(\alpha) \bar{\lambda}(\alpha, c(\alpha))=h(c(\alpha))$ at $\alpha_{0}$. Since $d c / d \alpha$ is positive at $\alpha_{0}$, it follows that (8) is true if and only if

$$
h^{\prime}\left(c_{0}\right)<0 .
$$

Hence a solution $c_{0}$ to (7) making (9) true gives a Hopf bifurcation in $\mathscr{H}$. Since $h^{\prime}(c)=k c f^{\prime \prime}(c)-(1-k) f^{\prime}(c)$, it is clear that the existence of the bifurcation only depends on the feedback function $f, f^{\prime}$, and $f^{\prime \prime}$. We have the following theorem: 
THEOREM. Suppose $n=5$ and $\alpha_{i}=\alpha$ in (1). Let $h(c)=k c f^{\prime}(c)-f(c)$. If there is a $c_{0}>0$ such that $h\left(c_{0}\right)=0$ and $h^{\prime}\left(c_{0}\right)<0$, then for $\alpha_{0} \equiv\left(f\left(c_{0}\right) / c_{0}\right)^{1 / 5}$ the vector field $F_{\alpha}$ of (1) has a Hopf bifurcation when $\alpha=\alpha_{0}$ at the critical point corresponding to $c_{0}$.

3. Griffith model. For $f$ given by (2) we have that $f^{\prime}(c)=p c^{p-1} /\left(1+c^{p}\right)^{2}$. Thus

$$
h(c)=c^{p}\left(k p-1-c^{p}\right) /\left(1+c^{p}\right)^{2} .
$$

There is a $c_{0}>0$ so that $h\left(c_{0}\right)=0$ if and only if $k p-1>0$. But $k p-1>0$ if and only if $p>1 / k$. And, in this case, $c_{0}=(k p-1)^{1 / p}$. A computation shows that

$$
h^{\prime}\left(c_{0}\right)=-p c_{0}^{2 p-1} /\left(1+c_{0}^{p}\right)^{2} .
$$

Hence $h^{\prime}\left(c_{0}\right)<0$. The lower bound of $1 / k$ for $p$ is approximately 305 . A $p$-value of 305 is probably too large to be biochemically feasible.

We summarize the critical point bifurcations of (1) as $\alpha$ changes. For large $\alpha$, the origin is the only critical point of (1) (see Fig. 1), and it is globally attracting. As $\alpha$ decreases, a degenerate critical point appears in $\mathscr{H}$ and bifurcates into an unstable and a stable critical point. This unstable critical point has a one-dimensional unstable manifold. When $\alpha$ decreases through $\alpha_{0}$, two eigenvalues of the unstable critical point cross through the imaginary axis in the positive-real direction. Hence, at least one periodic orbit appears near the unstable critical point. As $\alpha$ decreases to zero, the unstable critical point approaches the origin. When $\alpha=0$, the origin is a repeller in $\mathscr{H}$. Since $\mathscr{H}$ is invariant for the positive-time solution flow, as $\alpha$ decreases to zero the Hopf periodic orbit either disappears or moves far away, in $\mathscr{H}$, from the unstable critical point.

4. Tyson-Othmer model. For $f$ given by (3) we have that $f^{\prime}(c)=p(K-1) c^{p-1} /$ $\left(K+c^{p}\right)^{2}$ and so

$$
h(c)=-\left(c^{2 p}+(K+1+k p-k p K) c^{p}+K\right) /\left(K+c^{p}\right)^{2} .
$$

The numerator in (10) is quadratic in $c^{p}$. Thus $h$ has a positive zero, $c_{0}$, if and only if the numerator in (10) has a positive root $c_{0}^{p}$. This numerator has a positive root if both the following inequalities are satisfied:

$$
\begin{gathered}
-(K+1+k p-k p K)>0, \\
(K+1+k p-k p k)^{2}-4 K>0 .
\end{gathered}
$$

Eq. (11) is true if $p>(K+1) / k(K-1)$; and, assuming (11) is true, (12) is true if $p>(\sqrt{ } K+1) / k(\sqrt{ } K-1)$. Since $K>1$, the second lower bound for $p$ is larger than the first. Hence, if $p>(\sqrt{ } K+1) / k(\sqrt{ } K-1)$, we take the larger root of the numerator in (10) to get $c_{0}$ where $h\left(c_{0}\right)=0$.

A computation gives

$$
h^{\prime}\left(c_{0}\right)=-p c_{0}^{p-1}\left(2 c_{0}^{p}+(K+1+k p-k p K)\right) /\left(K+c_{0}^{p}\right)^{2} .
$$

Since $2 c_{0}^{p}>-(K+1+k p-k p K), h^{\prime}\left(c_{0}\right)<0$. Hence a Hopf bifurcation occurs for this $c_{0}$. Notice that the lower bound for $p$ in the Tyson-Othmer model is larger than the lower bound for $p$ in the Griffith model. 


\section{REFERENCES}

[1] W. A. Coppel, Stability and asymptotic behavior of differential equations, D. C. Heath, Boston, 1965

[2] J. S. Griffith, Mathematics of cellular control processes, II: Positive feedback to one gene, J. Theor. Biol. 20, 209-216 (1968)

[3] M. W. Hirsch, Systems of differential equations that are competitive or cooperative, II. Convergence almost everywhere, to appear

[4] J. E. Marsden and M. McCracken, The Hopf bifurcation and its applications, Springer-Verlag, New York, 1976

[5] J. Selgrade, Mathematical analysis of a cellular control process with positive feedback, SIAM J. Appl. Math. 36, 219-229(1979)

[6] —-, Asymptotic behavior of solutions to single loop positive feedback systems, J. Diff. Eq. 38, 80-103 (1980)

[7] J. J. Tyson and H. G. Othmer, The dynamics of feedback control circuits in biochemical pathways, Progr. Theor. Biol. 5, 1-62 (1978) 